\title{
2 Hydrophobic bioadhesive composites for human motion detection
}

3 Manisha Singh ${ }^{1,2}$, Ivan Solic ${ }^{2}$, Terry W. J. Steele ${ }^{1,2^{*}}$

$4 \quad{ }^{1}$ NTU-Northwestern Institute for Nanomedicine (NNIN), Interdisciplinary Graduate School

5 (IGS), Nanyang Technological University (NTU), Singapore 637553

$6{ }^{2}$ School of Materials Science and Engineering (MSE), Nanyang Technological University

7 (NTU), Singapore 639798

$8 \quad{ }^{*}$ Correspondence to Terry W. J. Steele (e-mail: wjsteele@ntu.edu.sg)

\section{CaproGlu Synthesis}

4-[3-(Trifluoromethyl)-3H-diazirin-3-yl] benzoic acid (230.14 g.mol-1 Dz-COOH) was synthesized from Dz-CH2OH. The solution of Dz-CH2OH (1 mol. eq.) and potassium hydroxide (1.55 mol. eq.; 0.6 M KOH solution) was dissolved in a mixture of water/dioxane $(5 / 1)$, cooled down to $0{ }^{\circ} \mathrm{C}$ in an ice bath and potassium permanganate (1.5 eq.) was added portion-wise. The biphasic reaction mixture was vigorously stirred at room temperature overnight (18 hours). The suspension was filtered through a pad of Celite ${ }^{\circledR}$. The filtrate was cooled in an ice bath and acidified with $2 \mathrm{M} \mathrm{HCl}$ to $\mathrm{pH}=1$. The obtained white precipitate was filtered off and dissolved in ethanol. The organic layer was washed 5 times with water and one time with brine, dried with anhydrous $\mathrm{MgSO} 4$, and the solvent was evaporated in a vacuum to yield pure Dz-COOH as a pale-yellow solid. The Proprietary CaproGlu synthesis method was the esterification reaction between PCLT (polyol) and Dz-COOH (acid) with 1,1carbonyldiimidazole (CDI) used as a coupling agent. The molar ratio of Dz-COOH/PCLT = $2 / 1$ was deliberately chosen to yield $\sim 50 \%$ diazirine conjugation. The reactants were placed in a round-bottomed flask with a magnetic stir bar. The flask was immersed in a water bath placed on the top of the heater equipped with a magnetic stirrer. DCM was added dropwise and stirred at room temperature for $1 \mathrm{~h}$. The solution of PCLT in DCM was added into the reaction mixture slowly and the round bottom flask was fitted with a condenser. The reaction mixture was heated to $40{ }^{\circ} \mathrm{C}$ and stirred for $18 \mathrm{~h}$. After completion DCM was removed on rotavap and the product CaproGlu was purified by water $/ \mathrm{Et}_{2} \mathrm{O}$ extraction (the water phase contained saturated $\mathrm{NaHCO}_{3}$ to remove unreacted $\mathrm{Dz}-\mathrm{COOH}$ ). The organic layer was dried with anhydrous $\mathrm{MgSO}_{4}$ 
and filtrated. $\mathrm{Et}_{2} \mathrm{O}$ was removed with rotavap. Traces of solvent from CaproGlu product were removed on a high vacuum for $6 \mathrm{~h}$ at $40{ }^{\circ} \mathrm{C}$.

\section{Technical details on the CNTs, as provided by the manufacturer ${ }^{1}$ :}

\begin{tabular}{|l|l|}
\hline Properties of CNT & Value \\
\hline Average diameter & $9.5 \times 10^{-9} \mathrm{~m}$ \\
\hline Average length & $1.5 \mu \mathrm{m}$ \\
\hline Carbon purity & $90 \%$ \\
\hline Surface Area & $250-300 \mathrm{~m}^{2} / \mathrm{g}$ \\
\hline Volume resistivity Technical Data Sheet (DM-TI-02-TDS-NC7000-V08). \\
\hline $\begin{array}{l}{ }^{1} \mathrm{NC7000} \\
\text { https://www.nanocyl.com/download/tds-nc7000/ }\end{array}$ \\
\hline
\end{tabular}
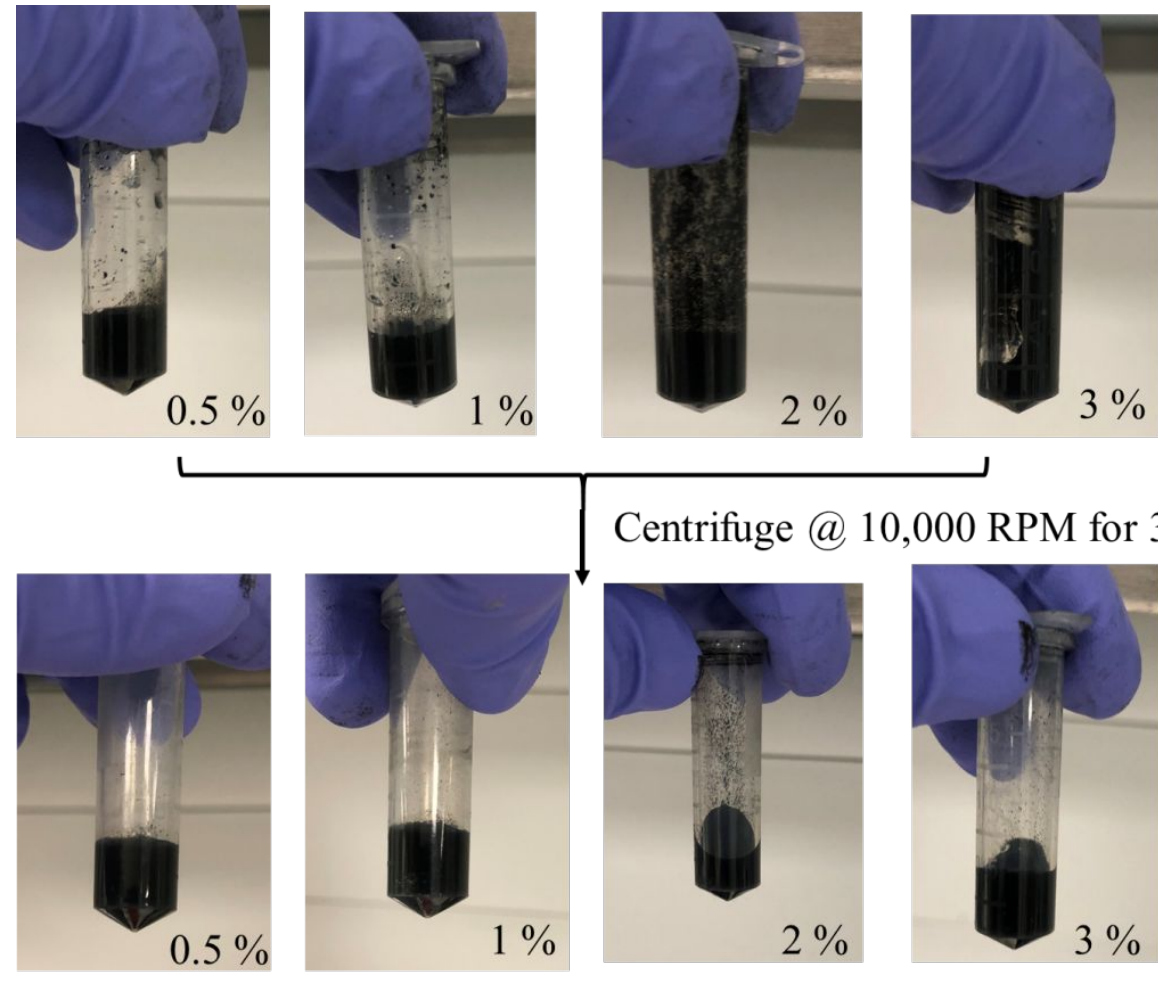

Centrifuge@10,000 RPM for 3 mins
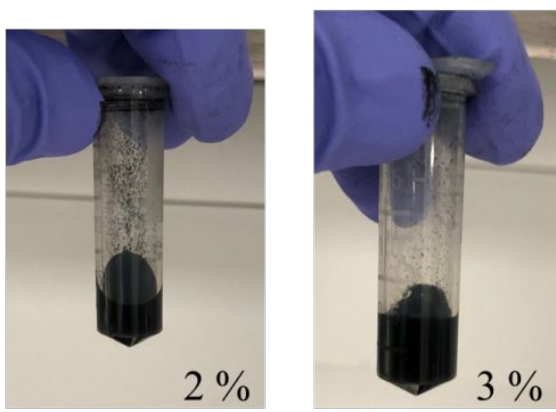

Figure S1: CNTs were physically mixed and remained dispersed in CaproGlu even after centrifuging at 10000 RPM for 3 minutes. 


\section{Scanning Electron Microscopy (SEM)}

42 For qualitative analysis of CNTs dispersion in the UV-cured CaproGlu matrix, SEM analyzed 43 the morphology of the composites. Cured samples were subjected to platinum coating $(90 \mathrm{~s}$, 44 chamber pressure $<5 \mathrm{~Pa}$ at $20 \mathrm{~mA}$ ). Images were obtained at an acceleration voltage of $5 \mathrm{kV}$ 45 and a working distance of 8-9 mm (Figure S4).
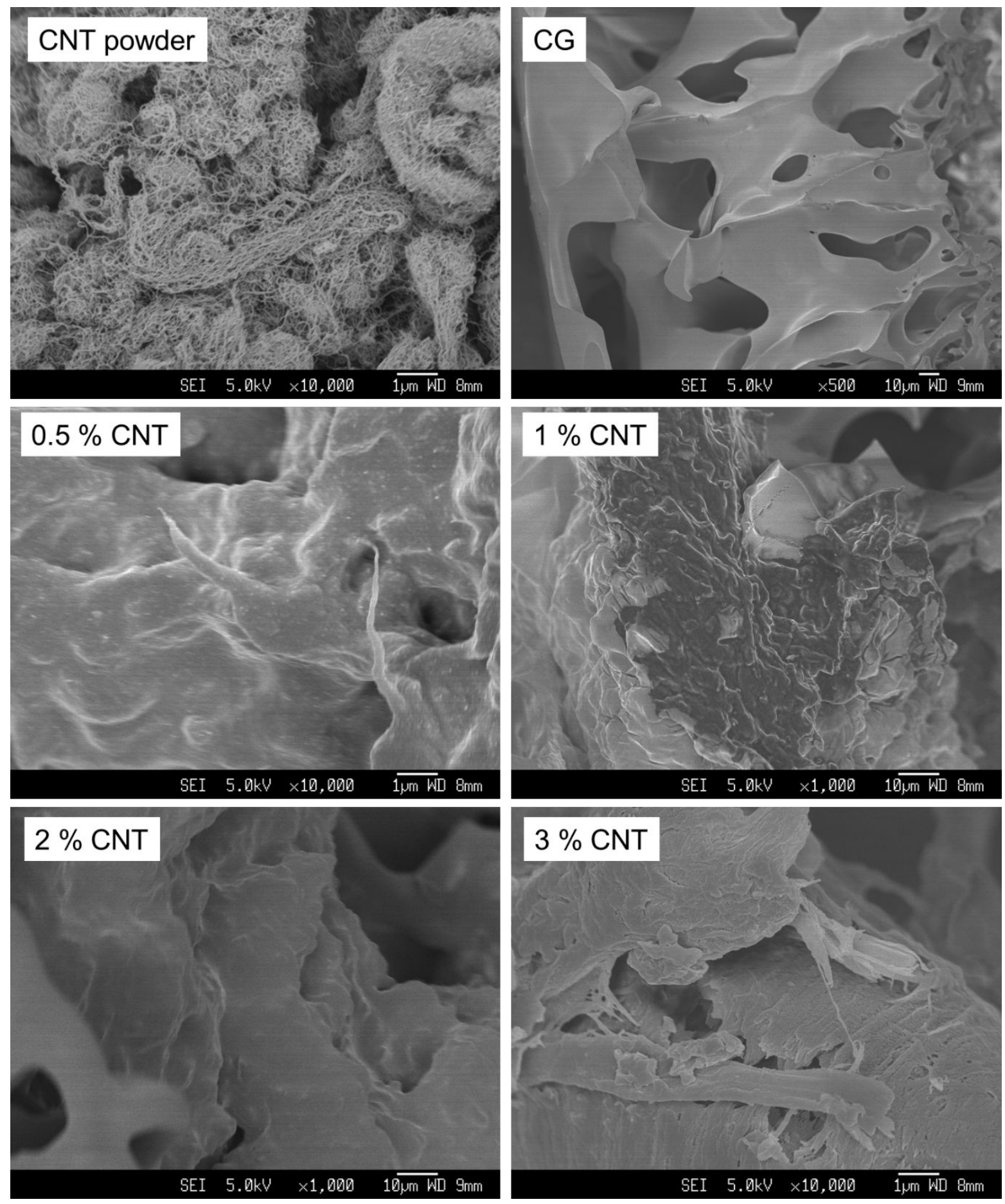
47 Figure S2: Cross-sectional morphology of CaproGlu composites under SEM. The surface of 48 CaproGlu control (CG) seems smooth, whereas the cross-sectional morphology of the composites shows rough-textured dispersion of CNTs in the matrix.

50
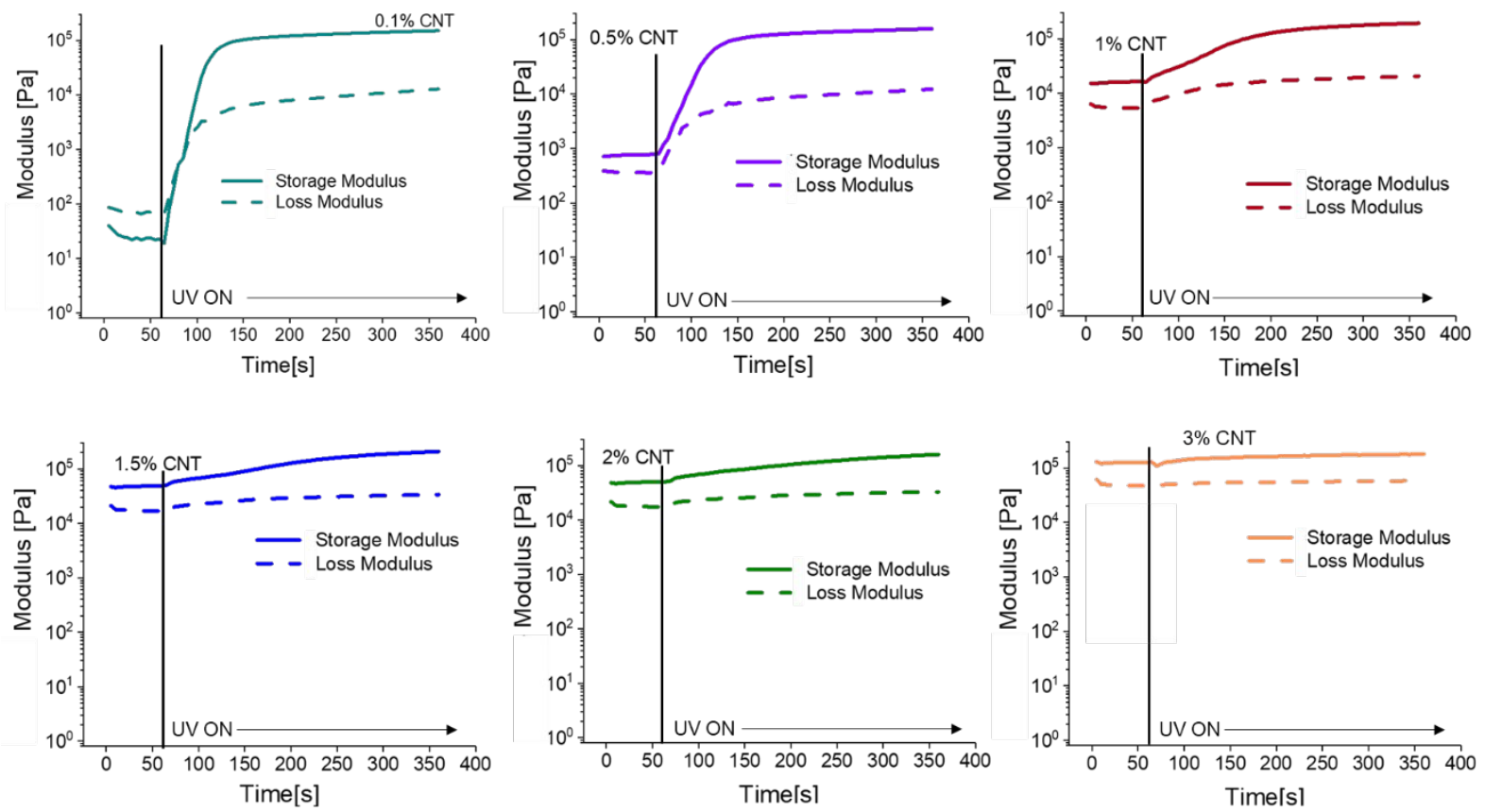

52 Figure S3: Real-time stimuli-based oscillatory rheology for CaproGlu composites showing G' and G'.

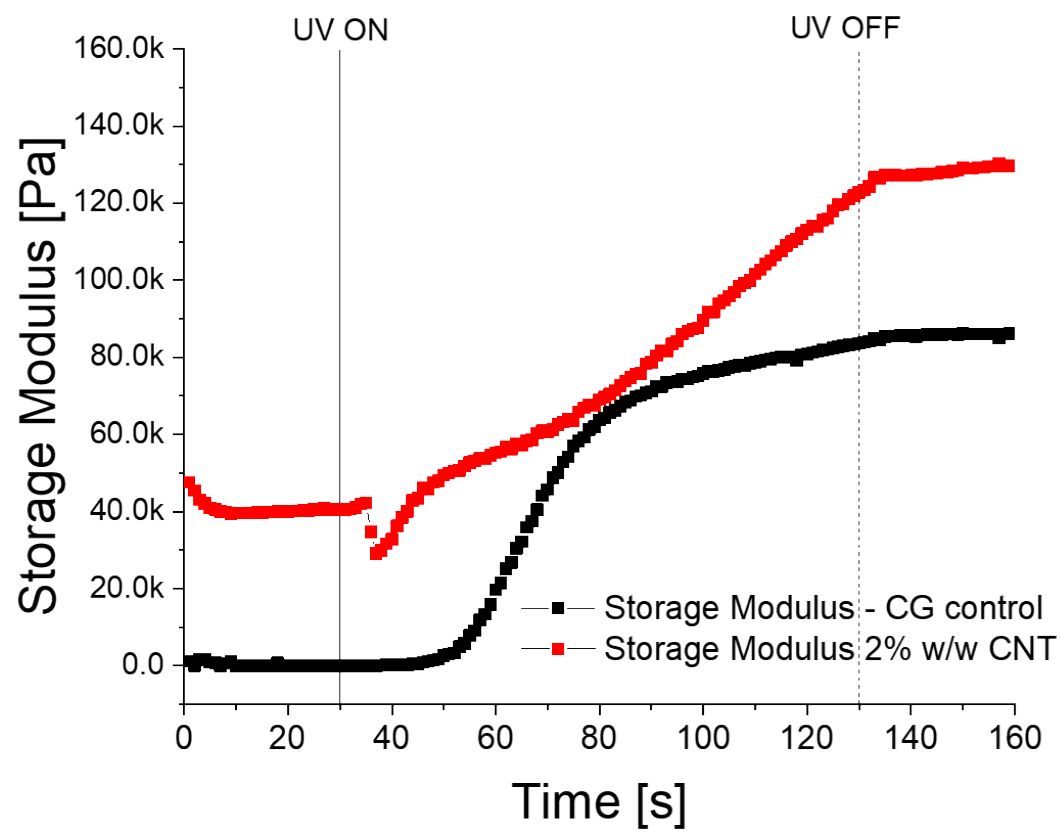

55 Figure S4: Real-time stimuli-based oscillatory rheology for CaproGlu composites showing G' stabilizes once UV is turned off. 
57 Mechanical, adhesion, and electrical properties of the composites under thermal curing

(A)

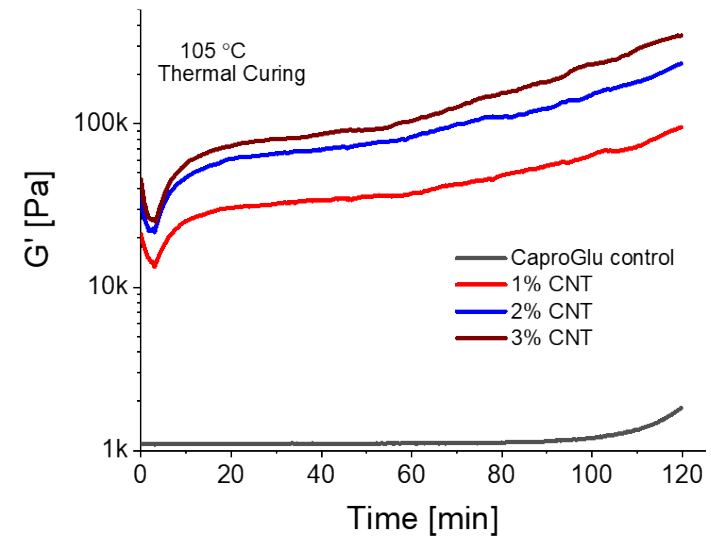

(B)

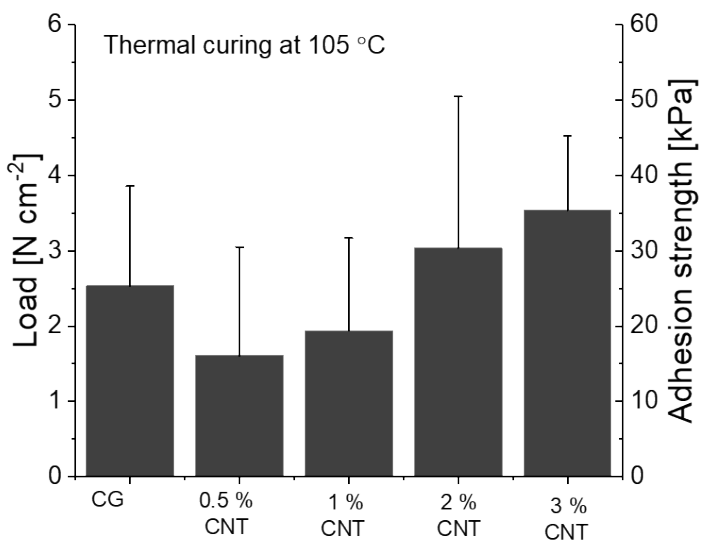

(C)

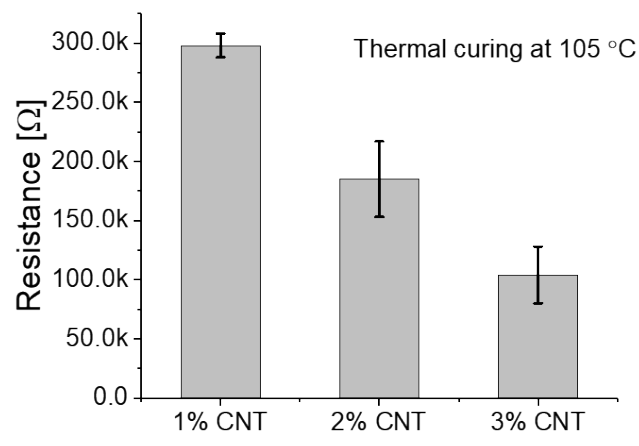

59

60 Figure S5: Evaluation of CaproGlu composites under thermal curing at $105{ }^{\circ} \mathrm{C}$. (A) 61 Comparison of real-time stimuli-based oscillatory rheology $\left(\mathrm{G}^{\prime}\right)$ for CaproGlu composites. (B) 62 Comparison of maximum lap-shear adhesion strength at a strain rate of $3 \mathrm{~mm} \mathrm{~min}^{-1}$. (C) 63 Comparison of resistance measured by the two-point probe method. 